TRANSACTIONS OF THE

AMERICAN MATHEMATICAL SOCIETY

Volume 182, August 1973

\title{
A WEDDERBURN THEOREM FOR ALTERNATIVE ALGEBRAS WITH IDENTITY OVER COMMUTATIVE RINGS
}

\author{
BY
}

W. C. BROWN

\begin{abstract}
In this paper, we study alternative algebras $\Lambda$ over a commutative, associative ring $R$ with identity. When $\Lambda$ is finitely generated as an $R$-module, we define the radical $J$ of $\Lambda$. We show that matrix units and split Cayley algebras can be lifted from $\Lambda / J$ to $\Lambda$ when $R$ is a Hensel ring. We also prove the following Wedderburn theorem: Let $\mathrm{\Lambda}$ be an alternative algebra over a complete local ring $R$ of equal characteristic. Suppose $\Lambda$ is finitely generated as an $R$-module, and $\Lambda / J$ is separable over $\bar{R}(\bar{R}$ the residue class field of $R)$. Then there exists an $\bar{R}$-subalgebra $S$ of $\mathbf{\Lambda}$ such that $S+J=\mathbf{\Lambda}$ and $S \cap J=0$.
\end{abstract}

Introduction. Let $\Lambda$ denote an alternative algebra over a field $R$. Let $J$ denote the radical of $\Lambda$. It is well known that the Wedderburn theorem holds for alternative algebras. That is, if $\Lambda$ is finite dimensional over $R$, and $\Lambda / J$ is separable over $R$, then there exists a separable subalgebra $S$ of $\Lambda$ such that $S \oplus J=$ $\Lambda$. If $\Lambda$ is associative, the author in [4] has generalized this result to the case where $R$ is a split Hensel ring. The purpose of this paper is to obtain similar results for alternative algebras over complete local rings $R$ of equal characteristic. The precise result is as follows: Let $R$ be a complete local ring of equal characteristic. Let $\bar{R}$ denote the residue class field of $R$. Let $\Lambda$ be an alternative algebra over $R$ such that $\Lambda$ is finitely generated as an $R$-module and, $\Lambda / J$ (J the radical of $\Lambda$ ) is separable over $R$. Then there exists an $\bar{R}$-subalgebra $S$ of $\Lambda$ such that $S+J=\Lambda$ and $S \cap J=0$.

In order to prove this result, we must carefully define what we mean by the radical $J$ of $\Lambda$. Once this has been done, the results follow much as in the as. sociative case.

Preliminaries. Throughout the rest of this paper, $R$ will denote an associative, commutative ring with identity $1 . \Lambda$ will always denote an alternative ring with identity. Thus, $\Lambda$ is a not necessarily associative or commutative ring which satisfies the following two identities:

Received by the editors June $30,1972$.

AMS (MOS) subject classifications (1970). Primary 17D05.

Key words and phrases. Alternative algebra, Hensel ring, complete local ring. 


$$
x^{2} y=x(x y), \quad y x^{2}=(y x) x \text { for all } x, y \in \Lambda
$$

It is not absolutely necessary to assume $\Lambda$ has an identity under multiplication. In the process of proving the main results of this paper, we could always first adjoin an identity to $\Lambda$. But for convenience we shall always assume $\Lambda$ contains an identity.

By the center $C(\Lambda)$ of $\Lambda$, we shall, as usual, mean the collection of all elements $c$ in $\Lambda$ which both commute and associate with all elements of $\Lambda$. We are now ready for the following definition: $\Lambda$ is an $R$-algebra if there exists a ring homomorphism $\theta: R \rightarrow C(\Lambda)$ such that $\theta(1)$ is the identity of $\Lambda$. From now on we shall suppress $\theta$ and simply write $r \lambda$ instead of $\theta(r) \lambda, r \in R$ and $\lambda \in \Lambda$. If $\Lambda$ is an $R$-algebra, then $\Lambda$ is naturally an $R$-module. In particular, an ideal $J \subset \Lambda$ is an $R$-submodule of $\Lambda$, i.e. $R J \subset J$. We say $\Lambda$ is free, finitely generated, flat etc. over $R$ if $\Lambda$ is free, finitely generated, flat etc. as an $R$-module. Throughout this paper, we assume that all ring homomorphisms of any rings in question which have identities take the identity to the identity. By an $R$-algebra homomorphism from $\Lambda$ to another alternative algebra $\Lambda^{\prime}$ over $R$, we shall mean an algebra homomorphism which is also an $R$-module homomorphism.

Many of the results which hold for alternative algebras over fields pass over to similar results in our setting. In particular, the Moufang identities [9, p. 28], Artin's theorem [9, Theorem 3.1] and the Peirce decomposition [9, PP. 32-37] hold for any alternative algebra $\Lambda$ over $R$. We shall use these results freely whenever needed.

Now let $R$ be a local ring, i.e. $R$ is a Noetherian ring with exactly one maximal ideal $m$. Let $\pi_{0}$ denote the natural projection of $R$ onto $\bar{R}=R / m$. We shall say that $R$ is split if there exists a ring homomorphism $\epsilon_{0}: \bar{R} \rightarrow R$ such that $\pi_{0} \epsilon_{0}$ is the identity map on $\bar{R}$. It follows from [6, Theorem 9] that any complete local ring of equal characteristic is split. If $R$ is split, then via $\epsilon_{0}$ we may identify $\bar{R}$ with a subring of $R$ containing 1 . If $\Lambda$ is a $n$ alternative algebra over a split local ring $R$, and $J$ is an ideal in $\Lambda$ containing $m \Lambda$, then $0 \rightarrow J \rightarrow \Lambda \rightarrow$ $\Lambda / J \rightarrow 0$ can naturally be viewed as a short exact sequence of $\bar{R}$-algebras. We shall say that this sequence splits if there exists an $\bar{R}$-algebra homomorphism $\epsilon: \Lambda / J \rightarrow \Lambda$ such that $\pi \epsilon$ is the identity map on $\Lambda / J$. Here $\pi$ is of course the natural projection of $\Lambda$ onto $\Lambda / J$.

Finally, we need the definition of a Hensel ring. Suppose $R$ is a local ring ( $R$ need not be Noetherian here) with maximal ideal $m$. Let $X$ be an indeterminate over $R$ and consider the polynomial ring $R[X]$. We have a natural ring homomorphism $\sigma: R[X] \rightarrow \bar{R}[X]$ induced by $\pi_{0}$. Namely if $f(X)=\Sigma r_{i} X^{i} \in R[X]$, then $\sigma(f)=\Sigma \pi_{0}\left(r_{i}\right) X^{i} \in \bar{R}[X]$. Let us write $\bar{f}$ for $\sigma(f)$. $R$ is called a Hensel ring if 
every monic polynomial $f(X) \in R[X]$ satisfies the following condition: If there exist two relatively prime polynomials $g_{1}(X)$ and $g_{2}(X) \in \bar{R}[X]$ such that $\bar{f}=g_{1} g_{2}$ and $g_{1}$ is monic, then there exist two polynomials $b_{1}(X)$ and $b_{2}(X) \in R[X]$ such that $b_{1} b_{2}=f, \bar{b}_{1}=g_{1}, \bar{b}_{2}=g_{2}$ and $b_{1}$ is monic. It is well known that complete local rings are Hensel rings [8, Theorem 30.4].

The author assumes the reader is familiar with the theory of associative algebras over Hensel rings [3].

I. The radical of an alternative algebra over a commutative ring. Let $\Lambda$ be an alternative algebra over $R$ which is finitely generated. Let $\Omega(R)$ denote the collection of all maximal ideals of $R$. Then for each $m \in \Omega(R), \Lambda / m \Lambda$ is an alternative algebra over the field $R / m$. Since $\Lambda$ is finitely generated as an $R$-module, $\Lambda / m \Lambda$ is finite dimensional over $R / m$. Thus, the radical of $\Lambda / m \Lambda$ is well defined and can be taken to be the collection of all properly nilpotent elements in $\Lambda / m \Lambda$ [9, Theorem 3.7]. Let $J(m)$ be the ideal in $\Lambda$ containing $m \Lambda$ and such that $J(m) / m \Lambda$ is the radical of $\Lambda / m \Lambda$. Thus, $J(m)$ is the full inverse image of the radical of $\Lambda / m \Lambda$ under the natural projection $\Lambda \rightarrow \Lambda / m \Lambda$. We now define the radical $J$ of $\Lambda$ as follows:

$$
J=\bigcap_{m \in \Omega(R)} J(m)
$$

We note that if $R$ is a field, then our definition of the radical of $\Lambda$ agrees with the classical definition for alternative algebras. If we assume $\Lambda$ is an associative algebra, then $J$ is just the Jacobson radical of $\Lambda$ [3, Corollary, p. 125]. In any case, we note that we have defined the radical of $\Lambda$ for only those alternative algebras which are finitely generated over $R$. We shall need the following facts about $J$.

Proposition 1. Let $\Lambda$ be an alternative algebra which is finitely generated over $R$. Let $J$ be the radical of $\Lambda$. Then if $x \in J, 1-x$ is a unit in $\Lambda$.

Proof. If $\Lambda$ has a generating set of cardinality $n$ over $R$, then for every $m \epsilon$ $\Omega(R)$ the dimension of $\Lambda / m \Lambda$ over $R / m$ is less than or equal to $n$. Hence by [9, Theorem 3.7], $J(\Lambda / m \Lambda)^{n}=0$. Thus, if $x \in J$, the image $\bar{x}$ of $x$ in $\Lambda / m \Lambda$ is nilpotent, i.e. $\bar{x}^{n}=0$ for any $m$. So,

Thus,

$$
(\overline{1}-\bar{x})\left(\overline{1}+\bar{x}+\cdots+\bar{x}^{n-1}\right)=\overline{1} \quad \text { in } \Lambda / m \Lambda \text {. }
$$

$$
1-(1-x)\left(1+x+\cdots+x^{n-1}\right) \in \bigcap_{m \in \mathbb{Q}(R)} m \Lambda \text {. }
$$

So, there is a $z \in \bigcap_{m} \Lambda$ such that $1=(1-x)\left(1+x+\cdots+x^{n-1}\right)+z$. Now if $\lambda \epsilon$ $\Lambda$, then $\lambda=\left[(1-x)\left(1+x+\cdots+x^{n-1}\right)\right] \lambda+z \lambda$. By Artin's theorem, $R[\lambda, x]$ is associative. Thus, $\left[(1-x)\left(1+x+\cdots+x^{n-1}\right)\right] \lambda=(1-x)\left(\lambda+\cdots+x^{n-1} \lambda\right)$. So as $R$-modules, we have $\Lambda=(1-x) \Lambda+m \Lambda$ for every $m \in \Omega(R)$. It now follows 
from Nakayama's lemma [3, Corollary, p. 124] that $\Lambda=(1-x) \Lambda$. So there exists a $\lambda^{1} \in \Lambda$ such that $(1-x) \lambda^{1}=1$. In a similar manner, we show $(1-x)$ has a left inverse. Therefore, $1-x$ has a unique two sided inverse.

An element $x \in \Lambda$ will be called quasi-regular if there exists an element $y \epsilon$ $\Lambda$ such that $x+y-x y=x+y-y x=0$. The element $y$ is called the quasi-inverse of $x$ and is easily seen to be unique. It follows from [10, Lemma 2] that $x$ is a quasi-regular if and only if $1-x$ is a unit in $\Lambda$. As in the associative theory, we say an ideal $I \subset \Lambda$ is quasi-regular if every element of $I$ is quasi-regular. It follows from Proposition 1 that $J$ is a quasi-regular ideal. We shall need the following proposition:

Proposition 2 (McCrimmon). Let $\Lambda$ be an alternative algebra, finitely generated over $R$. Let $J$ be the radical of $\Lambda$ and $e$ an idempotent in $\Lambda$. Then $J \cap$ $e \Lambda e=e J e$ is a quasi-regular ideal in the subalgebra $e \Lambda e$.

Proof. It follows easily from the Moufang identities that $e \Lambda e$ is a subalgebra of $\Lambda$. Since $J$ is an ideal in $\Lambda$, we have eJe $\subset J \cap e \Lambda e$. Let $x \in J \cap e \Lambda e$. Then $x=e \lambda e$ for some $\lambda \in \Lambda$. Now $e x=e(e \lambda e)=e \lambda e=x$. Similarily $x e=x$. Hence, $x=$ $e x e \in e J e$. So, $e J e=J \cap e \Lambda e$.

Suppose $x \in J \cap e \Lambda e$. Then $x \in J$. So, $x$ is a quasi-regular element in $\Lambda$. Let $y$ be the quasi-inverse of $x$ and consider eye $\epsilon e \Lambda e$. Using the Moufang identities, we have $x(e y e)=((x e) y) e=(x y) e=(x+y) e=x e+y e=x+y e$. If we now multiply by $e$, we get $e[x(e y e)]=x+e y e$. Now $x$ and eye $\epsilon e \Lambda e$. Therefore, $x(e y e) \epsilon$ $e \Lambda e$, and $e[x(e y e)]=x(e y e)$. Thus, eye is a right quasi-inverse for $x$. A similar argument shows eye is a left quasi-inverse for $x$. Thus, $x$ has a quasi-inverse eye in $e \Lambda e$. Since $x$ was an arbitrary element of $e J e$, we have shown $e$ Je is a quasi-regular ideal in $e \Lambda e$.

If $\Lambda$ is a finitely generated, associative algebra over $R$, and $x$ is a quasiregular element of $\Lambda$, then the quasi-inverse of $x$ is of the form $r_{1} x+r_{2} x^{2}+\cdots+$ $r_{n} x^{n}$ for $r_{i} \in R$. We shall need this same result for alternative algebras.

Proposition 3. Let $\Lambda$ be an alternative algebra, finitely generated over $R$. Let $x$ be a quasi-regular element of $\Lambda$ with quasi-inverse $y$. Then $y=r_{1} x+\cdots+$ $r_{n} x^{n}$ for some $n$ and $r_{i} \in R$.

Proof. Consider the subalgebra of $\Lambda$ generated by $x$ and $y$, i.e. $R[x, y]$. Since $y$ is the quasi-inverse of $x, y x=x y=x+y$. Thus, $R[x, y]$ is a commutative, associative ring. Since $\Lambda$ is finitely generated over $R$, it follows from [3, Theorem 8] that both $x$ and $y$ satisfy monic polynomials with coefficients in $R$. Thus, $R[x, y]$ is finitely generated as an $R$-module. The result now follows from applying [3, Theorem 9$]$ to $R[x, y]$. 
Finally we need to know how the radical behaves under homomorphic images.

Proposition 4. Let $\Lambda_{1}$ and $\Lambda_{2}$ be two alternative algebras over $R$ which are finitely generated. Let $J_{1}$ and $J_{2}$ be the radicals of $\Lambda_{1}$ and $\Lambda_{2}$ respectively. Suppose $\sigma: \Lambda_{1} \rightarrow \Lambda_{2}$ is an R-algebra epimorphism, then $\sigma\left(J_{1}\right) \subset J_{2}$.

Proof. For each $m \in \Omega(R)$, let $J_{i}(m)$ denote the pull back of the radical of $\Lambda_{i} / m \Lambda_{i}$ under the natural projection $\Lambda_{i} \rightarrow \Lambda_{i} / m \Lambda_{i}$. The map $\sigma$ induces an $R / m$ algebra homomorphism $\sigma_{m}: \Lambda_{1} / m \Lambda_{1} \rightarrow \Lambda_{2} / m \Lambda_{2}$ which is onto. So, we have the following commutative square of epimorphisms:

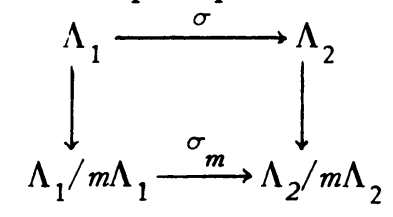

Since the radical of $\Lambda_{1} / m \Lambda_{1}$ consists of all properly nilpotent elements and $\sigma_{m}$ is onto, $\sigma_{m}$ takes the radical of $\Lambda_{1} / m \Lambda_{1}$ into the radical of $\Lambda_{2} / m \Lambda_{2}$. Since $J_{1}(m)$ is mapped onto the radical of $\Lambda_{1} / m \Lambda_{1}$, it follows that $\sigma\left(J_{1}(m)\right) \subset J_{2}(m)$. Since this holds for every $m \in \Omega(R)$, we have $\sigma\left(\bigcap_{m \in \Omega(R)} J_{1}(m)\right) \subset$ $\bigcap_{m \in \Omega(R)} J_{2}(m)$. Thus $\sigma\left(J_{1}\right) \subset J_{2}$.

II. Alternative algebras over Hensel rings. In this section, we shall assume that $R$ is a Hensel ring. This type of assumption is inevitable in trying to prove a Wedderburn type theorem because Hensel rings are the only commutative rings which permit idempotents and matrix units to be lifted from $\Lambda / J$ to $\Lambda$.

Theorem 1. Let $\Lambda$ be a finitely generated alternative algebra over a Hensel ring $R$. Let $I$ be an ideal in $\Lambda$. If $\bar{e}_{1}, \cdots, \bar{e}_{n}$ are pairwise orthogonal idempotents in $\Lambda / l$, then there exist pairwise orthogonal idempotents $e_{1}, \cdots, e_{n}$ in $\Lambda$ such that $\pi\left(e_{i}\right)=\bar{e}_{i}$. Here $\pi$ is the natural projection of $\Lambda$ onto $\Lambda / l$.

Proof. Suppose $\bar{e}$ is an idempotent in $\Lambda /$ l. Let $c \in \Lambda$ such that $\pi(c)=\bar{e}$. Set $S=R[c]$. Then $S$ is a commutative extension of $R$ which by [3, Theorem 8] is finitely generated as an $R$-module. $I \cap S$ is an ideal in $S$, and $S / I \cap S$ contains $\bar{e}$. Thus by [3, Theorem 24], $S$ contains an idempotent $e$ such that $\pi(e)=\bar{e}$. Hence, any idempotent in $\Lambda / I$ can be lifted to $\Lambda$.

Now suppose $e$ is an idempotent in $\Lambda$, and $\bar{e}_{1}$ is an idempotent in $\Lambda / 1$ such that $\bar{e} \bar{e}_{1}=\bar{e}_{1} \bar{e}=0 \quad(\bar{e}=\pi(e))$. Then there exists an idempotent $e_{1} \in \Lambda$ such that $e_{1} e=e e_{1}=0$, and $\pi\left(e_{1}\right)=\bar{e}_{1}$. The proof of this is as follows: Let $T(e)=\{x \in \Lambda \mid$ $x e=e x=0\}$, the set of two sided annihilators of $e$ in $\Lambda$. Suppose $x, y \in T(e)$. Then $(x y) e=(x, y, e)+x(y e)=(x, y, e)=-(x, e, y)=0$. Here $(x, y, z)=(x y) z-$ $x(y z)$ is the associator of three elements. Thus, $T(e)$ is a subalgebra of $\Lambda$ and clearly consists of all elements of the form $\lambda-e \lambda-\lambda e+e \lambda e, \lambda \epsilon \Lambda$. Hence 
$\pi(T(e))=\{\bar{x} \in \Lambda / I \mid \bar{x} \bar{e}=\bar{e} \bar{x}=0\}=T(\bar{e}) . \bar{e}_{1} \in T(\bar{e})$ by hypothesis. Thus, there exists an element $c \in T(e)$ such that $\pi(c)=\bar{e}_{1}$. Now consider $R\langle c\rangle$ the subalgebra of $\Lambda$ consisting of all polynomials in $c$ without constant term. Then $R\langle c\rangle c$ $T(e)$. It follows from [3, Theorem 21] that $R\langle c\rangle$ contains an idempotent $e_{1}$ such that $\pi\left(e_{1}\right)=\bar{e}_{1}$. Thus, $\Lambda$ contains an idempotent $e_{1}$ with $\pi\left(e_{1}\right)=\bar{e}_{1}$ and $e_{1} e=$ $0=e e_{1}$.

Now suppose $\bar{e}_{1}, \cdots, \bar{e}_{n}$ are pairwise orthogonal idempotents in $\Lambda / 1$. Lift $\bar{e}_{1}$ to an idempotent $e_{1} \in \Lambda$. By the second paragraph of this proof, lift $\bar{e}_{2}$ to an idempotent $e_{2}$ in $\Lambda$ orthogonal to $e_{1}$. Suppose we have lifted $\bar{e}_{1}, \cdots, \bar{e}_{m}, 1 \leq$ $m<n$, to pairwise orthogonal idempotents $e_{1}, \cdots, e_{m}$ in $\Lambda$. We can lift $\bar{e}_{m+1}$ to an idempotent $e_{m+1}$ in $\Lambda$ which is orthogonal to $e=\sum_{i=1}^{m} e_{i}$. Then for $i=1$, $\cdots, m$, we have $e_{i} e_{m+1}=\left(e e_{i} e\right) e_{m+1}=e\left[e_{i}\left(e e_{m+1}\right)\right]=0$. Similarly, $e_{m+1} e_{i}=0$. Thus, $e_{1}, \cdots, e_{m+1}$ are pairwise orthogonal, and the proof is completed by induction.

For future reference, we note that a slight modification of the proof of Theorem 1 yields the following result: Let $\Lambda$ be an alternative algebra over $R$ which is not necessarily finitely generated as an $R$-module. Suppose $I$ is an ideal in $\Lambda$ for which $I^{2}=0$. Then if $\bar{e}_{1}, \cdots, \bar{e}_{n}$ are pairwise orthogonal idempotents in $\Lambda / I$, there exist pairwise orthogonal idempotents $e_{1}, \cdots, e_{n} \in \Lambda$ such that $\pi\left(e_{i}\right)=$ $\bar{e}_{i}$. In this case, using the same $S$ and $R\langle c\rangle$ as appears in the proof of Theorem 1 , we note $I \cap S$ and $I \cap R\langle c\rangle$ are nilpotent ideals in $S$ and $R\langle c\rangle$ respectively. Thus, we may use the results in [7, Proposition 3.4, p. 42] to lift idempotents from $S / I \cap S$ to $S$ and from $R\langle c\rangle / I \cap R\langle c\rangle$ to $R\langle c\rangle$. Hence, Theorem 1 holds in general if $I^{2}=0$.

For the next proposition, we do not require $R$ to be a Hensel ring.

Proposition 5. Let $\Lambda$ be an alternative algebra over $R$. Let I be a quasiregular ideal in $\Lambda$. Let $e$ and $f$ be two pairwise orthogonal idempotents in $\Lambda$ whose images in $\Lambda / I$ we denote by $\bar{e}$ and $\bar{f}$. Suppose there exist elements $\bar{a} \epsilon$ $\bar{e}(\Lambda / I) \bar{f}$ and $\bar{b} \in \bar{f}(\Lambda / I) \bar{e}$ sucb that $\bar{a} \bar{b}=\bar{e}$ and $\bar{b} \bar{a}=\bar{f}$; then there exist elements $a \in e \Lambda f$ and $b \in f \Lambda e$ sucb that $a b=e, b a=f, \pi(a)=\bar{a}$, and $\pi(b)=\bar{b}$.

Here $\pi$ as usual denotes the natural projection of $\Lambda$ onto $\Lambda / l$.

Proof. It is well known that $(x, e, f)=0$ if $e$ and $f$ are pairwise orthogonal idempotents. Thus, $e \Lambda f$ and $f \Lambda e$ are unambiguous. Let $a_{1} \in e \Lambda f$ and $b_{1} \in f \Lambda e$ such that $\pi\left(a_{1}\right)=\bar{a}, \pi\left(b_{1}\right)=\bar{b}$. Then $a_{1}=e \lambda_{1} f$ and $b_{1}=f \lambda_{2} e$ for some $\lambda_{1}, \lambda_{2} \epsilon$ $\Lambda$. Thus, $a_{1} b_{1}=\left(e \lambda_{1} f\right)\left(f \lambda_{2} e\right)=e \cdot\left(\lambda_{1} f\right)\left(f \lambda_{2}\right) \cdot e \in e \Lambda e$. Also, $a_{1} b_{1}-e \in I$. Thus, $e-a_{1} b_{1} \in I \cap e \Lambda e$ which by the proof of Proposition 2 is a quasi-regular ideal in $e \Lambda e$. Since $e-a_{1} b_{1}$ is quasi-regular in $e \Lambda e, e-\left(e-a_{1} b_{1}\right)=a_{1} b_{1}$ is a unit in $e \Lambda e$. Thus, there exists an $x \in e \Lambda e$ such that $\left(a_{1} b_{1}\right) x=e=x\left(a_{1} b_{1}\right)$. Similarly, there exists a $y \in f \Lambda f$ such that $y\left(b_{1} a_{1}\right)=f=\left(b_{1} a_{1}\right) y$. 
Set $a=a_{1}$ and $b=b_{1} x$. Then $a \in e \Lambda /$. Since $x=e \lambda_{3} e$, for some $\lambda_{3} \in \Lambda$, to show $b \in f \Lambda e$ we must show $\left(f \lambda_{2} e\right)\left(e \lambda_{3} e\right) \in f \Lambda e$. Now $f\left[\left(f \lambda_{2} e\right)\left(e \lambda_{3} e\right)\right]=$ $-\left(f, f \lambda_{2} e, e \lambda_{3} e\right)+\left[f\left(f \lambda_{2} e\right)\right]\left(e \lambda_{3} e\right)$. By the Moufang identities, $\left(f, f \lambda_{2} e, e \lambda_{3} e\right)=$ $\left(f^{2}, f \lambda_{2} e, e \lambda_{3} e\right)=\left(f, f \lambda_{2} e, f\left(e \lambda_{3} e\right)+\left(e \lambda_{3} e\right) f\right)=0$ since $f\left(e \lambda_{3} e\right)=(f e) \cdot \lambda_{3} \cdot e=$ $0=e \cdot \lambda_{3} \cdot(e f)=\left(e \lambda_{3} e\right) f$. Also $f\left(f \lambda_{2} e\right)=f \lambda_{2} e$ by Artin's theorem. Hence, $f\left[\left(f \lambda_{2} e\right)\left(e \lambda_{3} e\right)\right]=\left(f \lambda_{2} e\right)\left(e \lambda_{3} e\right)$. Using the same techniques, we can show $\left[\left(f \lambda_{2} e\right)\left(e \lambda_{3} e\right)\right] e=\left(f \lambda_{2} e\right)\left(e \lambda_{3} e\right)$. Hence $b_{1} x=\left(f \lambda_{2} e\right)\left(e \lambda_{3} e\right) \in f \Lambda e$.

Now $a b=a_{1}\left(b_{1} x\right)=-\left(a_{1}, b_{1}, x\right)+e$. But $-\left(a_{1}, b_{1}, x\right)=\left(e \lambda_{1} f, e \lambda_{3} e, f \lambda_{2} e\right)=$ $\left(e \lambda_{1} f\right)\left(e \lambda_{3} e\right) \cdot\left(f \lambda_{2} e\right)-\left(e_{1} \lambda_{1} f\right) \cdot\left(e \lambda_{3} e\right)\left(f \lambda_{2} e\right)=\left[e \cdot\left(\lambda_{1} f\right)\left(e \lambda_{3}\right) \cdot e\right] \cdot\left(f \lambda_{2} e\right)-$ $\left(e \lambda_{1} f\right) \cdot\left[e \cdot\left(\lambda_{3} e\right)\left(f \lambda_{2}\right) \cdot e\right]=e \cdot\left[\left(\lambda_{1} f\right)\left(e \lambda_{3}\right) \cdot e\left(f \lambda_{2} e\right)\right]-\left[\left(e \lambda_{1} f\right) e \cdot\left(\lambda_{3} e\right)\left(f \lambda_{2}\right)\right] \cdot e=$ 0 . Thus $a b=e$.

In order to show $b a=f$, we first need $y b_{1}=b_{1} x$. Now the proof above shows $b_{1} a_{1} b_{1} \in f \Lambda e$. From this fact, one can easily argue that $\left(y, b_{1} a_{1} b_{1}, x\right)=0$ and $\left(y, b_{1}, a_{1}\right)=0$. These are routine arguments like the one above and will be omitted. We then have $y b_{1}=y\left(b_{1} e\right)=y \cdot\left[b_{1} \cdot\left(a_{1} b_{1}\right) x\right]$. But we have shown that $\left(a_{1}, b_{1}, x\right)=0$. So, $y\left(b_{1} e\right)=y \cdot\left[b_{1} \cdot\left(a_{1} b_{1}\right) x\right]=y \cdot\left(b_{1} \cdot a_{1}\left(b_{1} x\right)\right)=y \cdot\left(b_{1} a_{1} b_{1}\right) x=$ $y\left(b_{1} a_{1} b_{1}\right) \cdot x=\left(\left(y b_{1}\right) a_{1} \cdot b_{1}\right) \cdot x=\left(y\left(b_{1} a_{1}\right) \cdot b_{1}\right) \cdot x=\left(f b_{1}\right) x=b_{1} x$. Thus, $y b_{1}=$ $b_{1} x$. It now follows that $b a=\left(b_{1} x\right) a=\left(y b_{1}\right) a=y\left(b_{1} a\right)=f$.

To finish the proof, we note that $\pi(a)=\bar{a}$ and $\pi(b)=\bar{b}$.

We can now prove the main result of this section.

Theorem 2. Let $\Lambda$ be an alternative algebra over a Hensel ring $R$. Assume $\Lambda$ is finitely generated over $R$, and $I$ is a quasi-regular ideal in $\Lambda$. Suppose $\left\{\bar{e}_{i j} \mid i, j=1, \cdots, n\right\}$ is a system of matrix units in $\Lambda / I$, then there exists a system of matrix units $\left\{e_{i j} \mid i, j=1, \cdots, n\right\}$ in $\Lambda$ such that $\pi\left(e_{i j}\right)=\bar{e}_{i j}$.

Proof. Since $\bar{e}_{11}, \cdots, \bar{e}_{n n}$ are pairwise orthogonal idempotents in $\Lambda / l$, there exist, by Theorem 1 , pairwise orthogonal idempotents $e_{1}, \cdots, e_{n} \in \Lambda$ such that $\pi\left(e_{i}\right)=\bar{e}_{i i}$. Now for each $i=1, \cdots, n$, we have $\bar{e}_{11} \bar{e}_{1 i}=\bar{e}_{1 i}=\bar{e}_{1 i} \bar{e}_{i i}, \bar{e}_{i i} \bar{e}_{i 1}=$ $\bar{e}_{i 1}=\bar{e}_{i 1} \bar{e}_{11}$. Thus, $\bar{e}_{1 i} \in \bar{e}_{11}(\Lambda / I) \bar{e}_{i i}, \bar{e}_{i 1} \in \bar{e}_{i i}(\Lambda / I) \bar{e}_{11}$ and $\bar{e}_{1 i} \bar{e}_{i 1}=\bar{e}_{11}, \bar{e}_{i 1} \bar{e}_{1 i}=$ $\bar{e}_{i i}$. Applying Proposition 5, we get elements $e_{i 1} \in e_{i} \Lambda e_{1}$ and $e_{1 i} \in e_{1} \Lambda e_{i}$ such that $e_{i 1} e_{1 i}=e_{i}, e_{1 i} e_{i 1}=e_{1}, \pi\left(e_{1 i}\right)=\bar{e}_{1 i}$ and $\pi\left(e_{i 1}\right)=\bar{e}_{i 1}$. Put $e_{11}=e_{1}$ and $e_{i j}=e_{i 1} e_{1 j}$ for $i \neq 1, j \neq 1$. We shall show that $\left\{e_{i j} \mid i, j=1, \cdots, n\right\}$ are the required matrix units of $\Lambda$.

We first note that $e_{i j} \in e_{i} \Lambda e_{j}$ because $e_{i j}=e_{i 1} e_{1 j}=\left(e_{i} \lambda_{1} e_{1}\right)\left(e_{1} \lambda_{2} e_{j}\right)$ for elements $\lambda_{1}, \lambda_{2} \in \Lambda[9,3.17]$. A simple argument involving the Moufang identities shows $e_{i}\left[\left(e_{i} \lambda_{1} e_{1}\right)\left(e_{1} \lambda_{2} e_{j}\right)\right]=\left[\left(e_{i} \lambda_{1} e_{1}\right)\left(e_{1} \lambda_{2} e_{j}\right)\right] e_{j}=e_{i j}$. Thus, $e_{i j} \in e_{i} \Lambda e_{j}$. We need to show that $e_{i j} e_{j k}=e_{i k}$ for all $i, j, k=1, \cdots, n$.

Let us first show $e_{i j} e_{k l}=0$ if $k \neq j$. A linearized form of the Moufang identity $[9,3.5]$ is as follows: 


$$
y \cdot(x a) z+y \cdot(z a) x=(y x) a \cdot z+(y z) a \cdot x
$$

for all $x, y, z$ and $a \in \Lambda$. If we set $y=e_{i j}, x=e_{k}, a=e_{k l}$ and $z=e_{l}$ and use the fact that $e_{i j} \in e_{i} \Lambda e_{j}$, (4) becomes

$$
e_{i j} e_{k l}+e_{i j} \cdot\left(e_{l} e_{k l}\right) e_{k}=\left(e_{i j} e_{l}\right) e_{k l} \cdot e_{k} \cdot
$$

If $k=l$, then $e_{i j} e_{k l}=e_{i j} e_{k}=0$. Thus, without loss of generality we may assume $k \neq l$. In this case, (5) becomes

$$
e_{i j} e_{k l}=\left[\left(e_{i j} e_{l}\right) e_{k l}\right] e_{k} .
$$

If $l \neq j$, then (6) implies $e_{i j} e_{k l}=0$. Thus, we may assume $l=j$. In this case, (6) becomes

$$
e_{i j} e_{k j}=\left(e_{i j} e_{k j}\right) e_{k}
$$

If $k=i$, (7) implies $e_{i j} e_{i j}=\left(e_{i j} e_{i j}\right) e_{i}=e_{i j}\left(e_{i j} e_{i}\right)=0$. If $i=j, e_{i j} e_{k j}=e_{i} e_{k j}=0$. Thus, we have reduced the proof to showing that $e_{i j} e_{k j}=0$ when $i, j$ and $k$ are all distinct.

Since $i, j$ and $k$ are all distinct, we get $e_{i}\left(e_{i j} e_{k j}\right)=\left(e_{i j}, e_{i}, e_{k j}\right)+e_{i j} e_{k j}=$ $e_{i j} e_{k j}$. Thus, $e_{i j} e_{k j} \in e_{i} \Lambda$. Now $0=\left(e_{j} e_{i j}\right) e_{k j}=\left(e_{j}, e_{i j}, e_{k j}\right)+e_{j}\left(e_{i j} e_{k j}\right)$. Since $e_{i j} e_{k j} \in e_{i} \Lambda, e_{j}\left(e_{i j} e_{k j}\right)=0$. Thus, $0=\left(e_{j}, e_{i j}, e_{k j}\right)$. So, $0=\left(e_{i j}, e_{j}, e_{k j}\right)=$ $\left(e_{i j} e_{j}\right) e_{k j}-e_{i j}\left(e_{j} e_{k j}\right)=e_{i j} e_{k j}$. Therefore, in all cases $e_{i j} e_{k l}=0$ if $k \neq j$.

It remains to show that $e_{i j} e_{j k}=e_{i k}$. If $i=j$, we have $e_{i j} e_{j k}=e_{i} e_{i k}=e_{i k}$. If $k=j$, we have $e_{i j} e_{j k}=e_{i j} e_{j}=e_{i j}=e_{i k}$. Thus, we may assume $i \neq j$ and $j \neq k$. Now $e_{i j} e_{j k}=e_{i j}\left(e_{j 1} e_{1 k}\right)=-\left(e_{i j}, e_{j 1}, e_{1 k}\right)+\left(e_{i j} e_{j 1}\right) e_{1 k}$. Using the previous result, one easily argues that $\left(e_{i j}, e_{j 1}, e_{1 k}\right)=0$. So $e_{i j} e_{j k}=\left(e_{i j} e_{j 1}\right) e_{1 k}$.

Now $e_{i j} e_{j 1}=\left(e_{i 1} e_{1 j}\right) e_{j 1}=\left(e_{i 1}, e_{1 j}, e_{j 1}\right)+e_{i 1}\left(e_{1 j} e_{j 1}\right)$. Again one may argue that $\left(e_{i 1}, e_{1 j}, e_{j 1}\right)=0$. We thus get $e_{i j} e_{j 1}=e_{i 1}$. Thus, $e_{i j} e_{j k}=\left(e_{i j} e_{j 1}\right) e_{1 k}=$ $e_{i 1} e_{1 k}=e_{i k}$. Since $e_{i i}=e_{i}$ and $\pi\left(e_{i j}\right)=\bar{e}_{i j}$, the proof is complete.

The proof of Theorem 2 requires Theorem 1, Proposition 5 and well known identities for alternative rings. The proof of Proposition 5 does not require $\Lambda$ to be finitely generated over $R$. Any ideal $I$ in $\Lambda$ whose square is zero is clearly a quasi-regular ideal. Thus, using the remark following Theorem 1, we could prove the following analogue of Theorem 2: Let $\Lambda$ be an alternative algebra over $R$ which is not necessarily finitely generated. Let $I$ be an ideal in $\Lambda$ such that $I^{2}=0$. Then if $\left\{\bar{e}_{i j} \mid i, j=1, \cdots, n\right\}$ is a system of matrix units in $\Lambda / I$, there exists a system of matrix units $\left\{e_{i j}\right\} \in \Lambda$ such that $\pi\left(e_{i j}\right)=\bar{e}_{i j}$. We shall have use of this result in $\$ I I I$ of this paper.

Let $R_{2}$ denote the ring of all $2 \times 2$ matrices with coefficients in $R, R_{2}$ is naturally equipped with an involution $x \rightarrow \bar{x}$ satisfying $x+\bar{x} \in R$ and $x \bar{x} \in R$. Namely, if $x=\left(\begin{array}{ll}a & b \\ c & d\end{array}\right)$, then $\bar{x}=\left(\begin{array}{cc}d & -b \\ -c & a\end{array}\right)$. We shall say that an alternative algebra $\Lambda$ over $R$ is a split Cayley algebra if $\Lambda \cong R_{2} \oplus v R_{2}$ where $v$ is an element such that $v^{2}=1$, and $x v=v \bar{x}$ for all $x \in R_{2}$. 
Suppose $\Lambda$ is an alternative algebra over a local ring $R$. Let $m$ be the unique maximal ideal of $R$. If $\Lambda$ is finitely generated over $R$, then the radical $J$ of $\Lambda$ is well defined. Since $J \supset m \Lambda, \Lambda / J$ is naturally an $R / m$-algebra.

Theorem 3. Let $\Lambda$ be an alternative algebra over a Hensel ring $R$. Suppose $\Lambda$ is finitely generated over $R$, and $\Lambda / J$ is a split Cayley algebra over $R / m$. Then there exists a split Cayley algebra $\Lambda_{0}$ over $R$ contained in $\Lambda$ such that $\pi\left(\Lambda_{0}\right)=\Lambda / J$.

Proof. In this proof, we denote $\pi(x)$ by $[x]$ for $x \in \Lambda$. By hypothesis, $\Lambda / J=$ $(R / m)_{2} \oplus[\omega](R / m)_{2}$ is a split Cayley algebra over the field $R / m$. Now by Theorem 2 , there exist matrix units $\left\{e_{i j} \mid i, j=1,2\right\}$ in $\Lambda$ such that $(R / m)_{2}=$ $\sum_{i, j=1}^{2}(R / m)\left[e_{i j}\right]$. Set $e=e_{11}+e_{22}$. Then $[e]=1$ the identity element of $\Lambda / J$. Thus, $1-e \in J$. By Proposition $1, e$ is a unit in $\Lambda$. So, there exists a $z \in \Lambda$ such that $e z=z e=1$. Since $R[z, e]$ is an associative subalgebra of $\Lambda$, we have $0=$ $[(1-e) e] z=(1-e)(e z)=1-e$. Thus $e_{11}+e_{22}=1$.

The proof from this point on is similar to $[9,3.21]$. We shall borrow freely from this result. Find $f_{i j}(i \neq j) \in \Lambda$ such that $\left[f_{i j}\right]=[\omega]\left[e_{j j}\right]$. We may assume $f_{i j} \in e_{i i} \Lambda e_{j j}$. Also $e_{j i} f_{i j}=c_{j} \in J \cap \Lambda_{j j}$. Here $\Lambda_{11}, \Lambda_{12}, \Lambda_{21}$ and $\Lambda_{22}$ are the Peirce spaces arising from the idempotents $e_{11}$ and $e_{22}$ in $\Lambda$. Set $b_{i j}=f_{i j}-e_{i j} c_{j}$ for $i \neq j$. Then $b_{i j} \in \Lambda_{i j},\left[b_{i j}\right]=\left[f_{i j}\right]$ and $e_{j i} b_{i j}=b_{i j} e_{j i}=0$ for $i \neq j$. We also have $\left[b_{i j}\right]\left[b_{j i}\right]=\left[f_{i j}\right]\left[f_{j i}\right]=\left[e_{i i}\right]$. Thus, $b_{i j} b_{j i}=e_{i i}-a_{i}$ for some element $a_{i} \in J \cap \Lambda_{i i}$. Now $\Lambda_{i i}=e_{i i} \Lambda e_{i i}$; and, by Proposition $2, J \cap \cap \Lambda_{i i}$ is a quasi-regular ideal in $\Lambda_{i i}$. Thus, there exists an element $a_{i}^{\prime} \in \Lambda_{i i}$ such that $\left(e_{i i}-a_{i}\right)\left(e_{i i}-a_{i}^{\prime}\right)=e_{i i}=$ $\left(e_{i i}-a_{i}^{\prime}\right)\left(e_{i i}-a_{i}\right)$. Furthermore, Proposition 3 implies that $a_{i}^{\prime}$ is a polynomial in $a_{i}$ without constant term. Now set

$$
p_{12}=\left(e_{11}-a_{1}^{\prime}\right) b_{12} \text { and } p_{21}=b_{21} \text {. }
$$

We note that $p_{12} \in \Lambda_{12}, p_{21} \in \Lambda_{21},\left[p_{12}\right]=\left[b_{12}\right]=\left[f_{12}\right]$ and $\left[p_{21}\right]=\left[b_{21}\right]=$ $\left[f_{21}\right]$. We wish to show that $p_{i j} p_{j i}=e_{i i}$ for $i \neq j$. Now $p_{12} p_{21}=\left[\left(e_{11}-a_{1}^{\prime}\right) b_{12}\right] b_{21}$. These elements have the form $e_{11}-a_{1}^{\prime}=e_{11} \lambda_{1} e_{11}, b_{12}=e_{11} \lambda_{2} e_{22}$ and $b_{21}=$ $e_{22} \lambda_{3} e_{11}$ for some $\lambda_{1}, \lambda_{2}$ and $\lambda_{3} \in \Lambda$. From this, one can easily argue that $\left(e_{11}-a_{1}^{\prime}, b_{12}, b_{21}\right)=0$. Thus, $p_{12} p_{21}=\left(e_{11}-a_{1}^{\prime}\right)\left(b_{12} b_{21}\right)=\left(e_{11}-a_{1}^{\prime}\right)\left(e_{11}-a_{1}\right)=$ $e_{11}$. To show that $p_{21} p_{12}=e_{22}$, we need the following identities,

$$
\begin{aligned}
& a_{i} b_{i j}=b_{i j} a_{j}, \\
& a_{i}^{\prime} b_{i j}=b_{i j} a_{j}^{\prime},
\end{aligned}
$$

For (9), we have $a_{i} b_{i j}=\left(e_{i i}-b_{i j} b_{j i}\right) b_{i j}=b_{i j}-\left(b_{i j} b_{j i}\right) b_{i j}=b_{i j}-b_{i j}\left(b_{j i} b_{i j}\right)=$ $b_{i j}-b_{i j}\left(e_{j j}-a_{j}\right)=b_{i j}-b_{i j}+b_{i j} a_{j}=b_{i j} a_{j}$. Therefore, (9) is proven.

(10) follows from (9) and the fact that $a_{i}^{\prime}$ is a polynomial in $a_{i}$. We first note that $\left(a_{i}, b_{i j}, a_{j}\right)=0$. Thus using (9), we have 


$$
\left(b_{i j} a_{j}\right)\left(e_{j i}-a_{j}\right)=\left(e_{i i}-a_{i}\right)\left(b_{i j} a_{j}\right) .
$$

Now $e_{i i}-a_{i}^{\prime} \in R e_{i i}\left[a_{i}\right] \subset \Lambda_{i i}$. Thus, $e_{i i}-a_{i}^{\prime}$ associates with $e_{i i}-a_{i}$ and $\left(b_{i j} a_{j}\right)$. Thus from (11), we get

$$
\left(e_{i i}-a_{i}^{\prime}\right)\left[\left(b_{i j} a_{j}\right)\left(e_{j j}-a_{j}\right)\right]=b_{i j} a_{j}
$$

One can now directly argue that $\left(e_{i i}-a_{i}^{\prime}, b_{i j} a_{j}, e_{j j}-a_{j}\right)=0$. Thus, multiplying both sides of (12) by $\left(e_{i i}-a_{i}^{\prime}\right)$, we get

$$
\left(e_{i i}-a_{i}^{\prime}\right)\left(b_{i j} a_{j}\right)=\left(b_{i j} a_{j}\right)\left(e_{j j}-a_{j}^{\prime}\right) .
$$

Since $a_{i}+a_{i}^{\prime}-a_{i}^{\prime} a_{i}=0$, we get $a_{i}^{\prime}=\left(-a_{i}\right)\left(e_{i i}-a_{i}^{\prime}\right)$. Thus using (9), (13) and the fact that $\left(e_{i i}-a_{i}^{\prime}\right)$ is a polynomial in $a_{i}$ with coefficients in $R e_{i i}$, we get

$$
\begin{aligned}
a_{i}^{\prime} b_{i j} & =\left[\left(-a_{i}\right)\left(e_{i i}-a_{i}^{\prime}\right)\right] b_{i j}=\left(e_{i i}-a_{i}^{\prime}\right)\left(-a_{i} b_{i j}\right)=\left(e_{i i}-a_{i}^{\prime}\right)\left(-b_{i j} a_{j}\right) \\
& =\left(-b_{i j} a_{j}\right)\left(e_{j j}-a_{j}^{\prime}\right)=b_{i j}\left[\left(-a_{j}\right)\left(e_{j j}-a_{j}^{\prime}\right)\right]=b_{i j} a_{j}^{\prime} .
\end{aligned}
$$

Therefore (10) is proven.

We can now prove $p_{21} p_{12}=e_{22}$. We first note that (9) and (10) imply $p_{12}=\left(e_{11}-a_{1}^{\prime}\right) b_{12}=b_{12}-a_{1}^{\prime} b_{12}=b_{12}-b_{12} a_{2}^{\prime}=b_{12}\left(e_{22}-a_{2}^{\prime}\right)$. Thus, $p_{21} p_{12}=$ $b_{21}\left[b_{12}\left(e_{22}-a_{2}^{\prime}\right)\right]=\left(b_{21} b_{12}\right)\left(e_{22}-a_{2}^{\prime}\right)=\left(e_{22}-a_{2}\right)\left(e_{22}-a_{2}^{\prime}\right)=e_{22}$.

We next note that $e_{i j} p_{j i}=p_{j i} e_{i j}=0$ if $i \neq j$. For $e_{12} p_{21}=e_{12} b_{21}=0$ and $e_{21} p_{12}=e_{21} \cdot\left(e_{11}-a_{1}^{\prime}\right) b_{12}=e_{21} b_{12} \cdot\left(e_{22}-a_{2}^{\prime}\right)=0$. Now set $v=p_{12}+p_{21}$. We note that $p_{i j} \in \Lambda_{i j}$, and thus $p_{i j}^{2}=0$ by $[9,3.20]$. So $v^{2}=\left(p_{12}+p_{21}\right)^{2}=p_{12} p_{21}+$ $p_{21} p_{12}=e_{11}+e_{22}=1$. We also have that $[v]=[w]$. Set $R_{2}=\Sigma_{i, j=1}^{2} R e_{i j} \subset \Lambda$. Then , $v \notin R_{2}$. One can easily show $v R_{2} \cap R_{2}=0$. Thus, we can consider $\Lambda_{0}=$ $R_{2} \oplus v R_{2} \subset \Lambda$. Clearly $\pi\left(\Lambda_{0}\right)=\Lambda / J$. So the theorem will be complete if we show that $x v=v \bar{x}$ for all $x \in R_{2}$.

Let $x \in R_{2}$. Then $x=a e_{11}+b e_{12}+c e_{21}+d e_{22}$ for constants $a, b, c$ and $d \epsilon$ $R$. Then $\bar{x}=d e_{11}-b e_{12}-c e_{21}+a e_{22}, x v=a p_{12}+b e_{12} p_{12}+c e_{21} p_{21}+d p_{21}$, and $v \bar{x}=a p_{12}-b p_{12} e_{12}-c p_{21} e_{21}+d p_{21}$. But if $x_{i j}, y_{i j} \in \Lambda_{i j}(i \neq j)$, then $x_{i j} y_{i j}=$ $-y_{i j} x_{i j}$ by $[9,3.21]$. Thus, $x v=v \bar{x}$.

In Theorem 3, if we replace $J$ by an ideal $I$ whose square is zero, then we could drop the hypothesis that $\Lambda$ is finitely generated over $R$. We would then have the following result: Suppose $\Lambda$ is an alternative algebra over $R$, and $I$ is an ideal in $\Lambda$ such that $I^{2}=0$. Suppose $\Lambda / I$ is a split Cayley algebra over $R / I \cap R$. Then there exists a split. Cayley algebra $\Lambda_{0}$ over $R$ contained in $\Lambda$ such that $\pi\left(\Lambda_{0}\right)=\Lambda / 1$. A proof of this remark would follow from the remark after Theorem 2 and the proof of [9, Lemma 3.21].

III. A Wedderburn theorem for alternative algebras over complete local rings of equal characteristic. Throughout this section, we assume $R$ is a split local ring. Thus, $\bar{R}=R / m$ is imbedded in $R$ via some ring monomorphism $\epsilon$. We shall drop $\epsilon$ and consider $\bar{R}$ as contained in $R$. 
Proposition 6. Let $\Lambda$ be an alternative algebra (not necessarily finitely generated) over a split local ring $R$. Suppose $I$ is an ideal in $\Lambda$ which contains $m \Lambda$ and has square zero. Then if $\Lambda / I$ is central separable over $\bar{R}, 0 \rightarrow I \rightarrow \Lambda \rightarrow$ $\Lambda / I \rightarrow 0$ splits as $\bar{R}$-algebras.

Proof. It is clearly sufficient to show that there exists an $\bar{R}$-subalgebra $S$ of $\Lambda$ such that $I \cap S=0$ and $I+S=\Lambda$. Since $\Lambda / I$ is central separable over the field $\bar{R}, \Lambda / I$ is either a Cayley algebra of dimension eight over $\bar{R}$, or $\Lambda / I$ is $n \times n$ matrices over an associative division algebra $D$. In the latter case, $D$ has center $\bar{R}$ and is finite dimensional over $\bar{R}$. In either case, let $K$ be a field which contains $\bar{R}$, is finite dimensional over $\bar{R}$ and splits $\Lambda / I$, i.e. $\Lambda / I \otimes{ }_{R} K$ is either a split Cayley algebra over $K$ or $n^{\prime} \times n^{\prime}$ matrices $K_{n}^{\prime}$ over $K$. Since $\Lambda / I$ is finite dimensional over $\bar{R}$, such a field $K$ exists. Write $K=\bar{R}\left(\epsilon_{1}, \cdots, \epsilon_{r}\right)$ with $\epsilon_{1}=1$.

Now consider $\Lambda \otimes_{\bar{R}} K$. This is an alternative algebra over the field $K$, and $I \otimes-K$ is an ideal nilpotent of index two in $\Lambda \otimes-K$. Furthermore, $\left(\Lambda \otimes_{\bar{R}}^{R} K\right) /\left(I \otimes_{\bar{R}} K\right) \cong(\Lambda / I) \otimes_{\bar{R}} K$. Thus, the remarks made after Theorems 2 and 3 when applied to $\Lambda \otimes_{\bar{R}} K$ imply that there exists a $K$-subalgebra $S^{\prime}$ of $\Lambda \otimes \otimes_{R} K$ such that $S^{\prime} \oplus\left(I \otimes_{\bar{R}} K\right)=\Lambda \otimes_{\bar{R}} K$. The proof now proceeds exactly as in the associative case $[1, \mathrm{PP} .47-48]$ to obtain an $\bar{R}$-subalgebra $S$ of $\Lambda$ with $S \oplus I=\Lambda$.

Proposition 7. Suppose $\Lambda$ is an alternative algebra (not necessarily finitely generated) over a split local ring $R$. Suppose $I$ is an ideal in $\Lambda$ which contains $m \Lambda$ and has square zero. Then if $\Lambda / I$ is separable over $\bar{R}, 0 \rightarrow I \rightarrow \Lambda \rightarrow \Lambda / I \rightarrow$ 0 splits as $\bar{R}$-algebras.

Proof. $\Lambda / I$ separable over $\bar{R}$ means $\Lambda / I$ is finite-dimensional over $\bar{R}$, and $\Lambda / I$ decomposes into a finite direct sum of ideals $S_{1} \oplus \ldots \oplus S_{n}$. Each $S_{i}$ is central simple over its center $Z_{i}$ which in turn is a separable field over $\vec{R}$. Let the identities of the subalgebras $S_{i}$ be $\bar{e}_{1}, \cdots, \bar{e}_{n}$. Then we can lift these pairwise orthogonal idempotents to pairwise orthogonal idempotents $e_{1}, \cdots, e_{n}$ in $\Lambda$. Since $\bar{e}_{1}+\cdots+\bar{e}_{n}=1$, it follows that $e_{1}+\cdots+e_{n}=1$ in $\Lambda$. We also know that $0 \rightarrow$ $e_{i} l e_{i} \rightarrow e_{i} \Lambda e_{i} \rightarrow S_{i} \rightarrow 0$ is exact. Thus, for the purposes of proving the proposition, we may assume $\Lambda / I$ is central simple over its center $Z$, and that $Z$ is a finite separable field extension of $\bar{R}$.

Hence, we may assume that $\Lambda / l$ is either $n \times n$-matrices over an associative division algebra $D$ having center $Z$, or that $\Lambda / I$ is a Cayley algebra over $Z$. In either case, since $Z$ is a finite separable extension of $\bar{R}$, there exists a field $F$ such that $F$ is a finite separable extension of $\bar{R}$, and $Z \otimes_{\bar{R}} F \cong \bigoplus_{i=1}^{l} \bar{e}_{i} F$ for pairwise orthogonal idempotents $\bar{e}_{1}, \cdots, \bar{e}_{l}$ in $Z \otimes_{\bar{R}} F$. Consider the exact sesequence of $F$-algebras: $0 \rightarrow I \otimes \otimes_{R} F \rightarrow \Lambda \otimes_{\bar{R}} F \rightarrow{ }^{\bar{R}} \Lambda / I \otimes_{\bar{R}} F \rightarrow 0$. If $\Lambda / I$ is as- 
sociative, then the center of $\Lambda / I \otimes_{\bar{R}} F$ is $Z \otimes_{\bar{R}} F$ [2, Corollary 1.6]. If $\Lambda / I$ is nonassociative, then $\Lambda / I$ is a Cayley algebra over $Z$. Thus, $\Lambda / I=Q \oplus v Q$ for some quaternian algebra $Q$ over $Z$. So $Q \otimes_{R} F$ is an associative subalgebra of $\Lambda / I \otimes_{\bar{R}} F$. Hence the center $C\left(\Lambda / I \otimes_{\bar{R}} F\right)$ of $\Lambda / I \otimes_{\bar{R}} F$ is contained in the center $C\left(Q \otimes_{\vec{R}} F\right)$ of $Q \otimes_{\bar{R}} F$. Now $Q$ is central separable over $Z$, and $Z$ is separable over $\bar{R}$. Thus, $Q$ is separable over $\bar{R}$. It again follows from [2, Corollary 1.6] that $C\left(Q \otimes_{\bar{R}} F\right)=Z \otimes_{\bar{R}} F$. Thus, in either case the center of $\Lambda / I \otimes_{\bar{R}} F$ is $Z \otimes \otimes_{R} F=\bigoplus_{i=1}^{l} F \bar{e}_{i}$.

Now from the remark after Theorem 1 , we may lift $\left\{\bar{e}_{1}, \cdots, \bar{e}_{l}\right\}$ to pairwise orthogonal idempotents $e_{1}, \cdots, e_{l}$ in $\Lambda \otimes_{R} F$ whose sum is 1 . For each $i=$ $1, \cdots, l, 0 \rightarrow e_{i}\left(I \otimes \otimes_{R} F\right) e_{i} \rightarrow e_{i}\left(\Lambda \otimes \otimes_{R} F\right) e_{i} \rightarrow\left(\Lambda / I \otimes \otimes_{R} F\right) \bar{e}_{i} \rightarrow 0$ is an exact sequence of $F$-algebras. $e_{i}\left(I \otimes \otimes_{R} F\right) e_{i}$ is a nilpotent ideal of index two, and the center of $\left(\Lambda / I \otimes \otimes_{\bar{R}} F\right) \bar{e}_{i}$ is isomorphic to $F$. Since $\Lambda / I \otimes_{\bar{R}} F$ is separable over $F,\left(\Lambda / I \otimes_{\bar{R}} F\right) \bar{e}_{i}^{R}$ is central separable over $F$. Thus, Proposition 6 implies there exists an $F$-subalgebra $S_{i}$ of $e_{i}\left(\Lambda \otimes{ }_{R} F\right) e_{i}$ such that $S_{i} \oplus e_{i}\left(I \otimes \frac{\otimes_{R}}{R}\right) e_{i}=$ $e_{i}\left(\Lambda \otimes_{R} F\right) e_{i}$. Setting $S^{\prime}=S_{1}+\cdots+S_{l}$, we get an $F$-subalgebra of $\Lambda \otimes_{R} F$ such that $\left(I \otimes_{R} F\right) \oplus S^{\prime}=\Lambda \otimes \frac{F}{R} F$. Following the proof of Wedderburn's theorem in [1, pp. 47-48], we can then find an $\bar{R}$-subalgebra $S$ of $\Lambda$ such that $S \oplus I=\Lambda$. This immediately implies $0 \rightarrow I \rightarrow \Lambda \rightarrow \Lambda / I \rightarrow 0$ splits.

We can now prove the main theorem.

Theorem 4. Let $\Lambda$ be an alternative algebra over a complete local ring $R$ of equal characteristic. Suppose $\Lambda$ is finitely generated as an $R$-module, and $\Lambda / J$ is separable over $\bar{R}=R / m$. Then there exists an $\bar{R}$-subalgebra $S$ of $\Lambda$ sucb that $S+J=\Lambda$ and $S \cap J=0$.

Proof. We had noted in the preliminaries that a complete local ring $R$ of equal characteristic contains a copy of its residue class field $\bar{R}$. We choose a copy of $\bar{R}$ in $R$ and regard $\Lambda$ as an $\bar{R}$-algebra via this copy.

For each $n \geq 1$, define $J^{2^{n}}=J^{2^{n-1}} J^{2^{n-1}}$. We first prove that $\Lambda$ is a complete Hausdorff space in its $J^{2^{n}}$-adic topology. By definition, $J$ is the complete inverse image of the radical of $\Lambda / m \Lambda$. Since $\Lambda$ is finitely generated as an $R$ module, $\Lambda / m \Lambda$ is a finite-dimensional alternative algebra. Hence, the radical of $\Lambda / m \Lambda$ is nilpotent. Therefore, there exists an $n_{0}>0$ such that $J^{2^{n} 0} \subset m \Lambda$. This

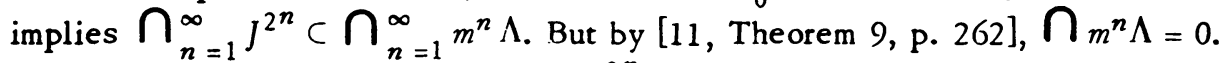
Thus, $\Lambda$ is a Hausdorff space in its $J^{2 n}$-adic topology. By [11, Theorem 5, p. 256], $\Lambda$ is a complete Hausdorff space in its $m \Lambda$-adic topology. Thus, $\Lambda$ is complete in its $J^{2^{n}}$-adic topology.

Now for each $n \geq 1$, we have 


$$
0 \rightarrow J / J^{2^{n}} \rightarrow \Lambda / J^{2^{n}} \rightarrow \Lambda / J \rightarrow 0
$$

is an exact sequence of alternative algebras. Since $\Lambda / J$ is separable over $\bar{R}$, one can easily argue, using Proposition 4, that $J / J^{2^{n}}$ is the radical of $\Lambda / J^{2^{n}}$. If $n=$ 1, Proposition 7 implies (14) splits as $\bar{R}$-algebras. Hence, there exists an $\bar{R}$-subalgebra $S_{2}$ of $\Lambda / J^{2}$ such that $S_{2} \oplus J / J^{2}=\Lambda / J^{2}$. In particular, $S_{2}$ is isomorphic to $\Lambda / J$. If $n=2$, we have the following commutative diagram with exact rows:

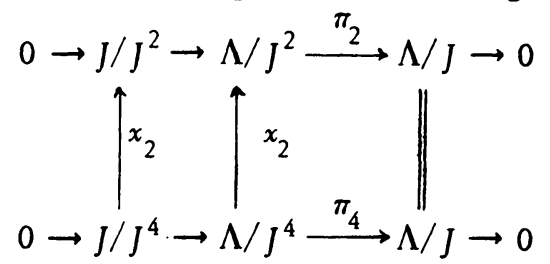

Here $x_{2}$ is the natural projection of $\Lambda / J^{4}$ onto $\Lambda / J^{2}$. Since $x_{2}$ is an $R$-algebra homomorphism, $x_{2}$ is also an $\bar{R}$-algebra homomorphism. Set $S_{4}^{\prime}=x_{2}^{-1}\left(S_{2}\right)$. Then $S_{4}^{\prime}$ is an $\bar{R}$-subalgebra of $\Lambda / J^{4}$. We note that

$$
0 \rightarrow J^{2} / J^{4} \rightarrow S_{4}^{\prime} \rightarrow S_{2} \cong \Lambda / J \rightarrow 0
$$

is an exact sequence of $\bar{R}$-algebras. Thus, by Proposition 7, (16) splits. Hence, there exists an $\bar{R}$-subalgebra $S_{4}$ of $\Lambda / J^{4}$ such that $S_{4} \oplus J^{2} / J^{4}=S_{4}^{\prime}$. Since $x_{2}\left(S_{4}\right)=S_{2}$, we have $\pi_{4}\left(S_{4}\right)=\Lambda / J$. From this it follows that $S_{4} \oplus J / J^{4}=\Lambda / J^{4}$. Thus (14) splits when $n=2$.

By induction, we can show that for each $n \geq 1$ there exists an $\bar{R}$-subalgebra $S_{2^{n}}$ of $\Lambda / J^{2^{n}}$ such that $S_{2^{n}} \oplus J / J^{2^{n}}=\Lambda / J^{2^{n}}$ and $x_{2^{n+1}}\left(S_{2^{n+1}}\right)=S_{2^{n}}$. Since $\Lambda$ is complete in its $J^{2^{n}}$-adic topology, the inverse limit $\lim \Lambda / J^{2^{n}}$ is isomorphic to $\Lambda$. If we set $S=\lim _{2^{n}}$ (see [11, pp. 305-306]), then $S^{-}$is an $\bar{R}$-subalgebra of $\Lambda$ for which $S \oplus J=\Lambda$ :

IV. A conjecture concerning Hensel rings. One may ask if there is a broader class of rings for which Theorem 4 holds. The completeness of $R$ was used heavily in the proof of Theorem 4, but Theorems 1, 2 and 3 seem to suggest that Theorem 4 may hold if $R$ is a split Hensel ring. The author conjectures that Theorem 4 holds if we assume $R$ is a split Hensel ring instead of $R$ being a complete local ring. In view of [5, Theorem], this would be the broadest class of local rings for which one could hope to prove Theorem 4.

\section{REFERENCES}

1. A. A. Albert, Structure of algebras, Amer. Math. Soc. Colloq. Publ., vol. 24, Amer. Math. Soc., Providence, R. I., 1939. MR 1, 99.

2. M. Auslander and $\mathrm{O}$. Goldman, The Brauer group of a commutative ring, Trans. Amer. Math. Soc. 97 (1960), 367-409. MR 22 \#12130.

3. G. Azumaya, On maximally central algebras, Nagoya Math. J. 2 (1951), 119-150. MR 12, 669 . 
4. W. C. Brown, Strong inertial coefficient rings, Michigan Math. J. 17 (1970), 7384. MR 41 \#8402.

5. W. C. Brown and E. C. Ingraham, A characterization of semilocal inertial coefficient rings, Proc. Amer. Math. Soc. 26 (1970), 10-14. MR 41 \#5354.

6. I. S. Cohen, On the structure and ideal theory of complete local rings, Trans. Amer. Math. Soc. 59 (1946), 54-106. MR 7, 509.

7. N. Jacobson, Structure of rings, Amer. Math. Soc. Colloq. Publ., vol. 37, Amer. Math. Soc., Providence, R. I., 1956. MR 18 \#373.

8. M. Nagata, Local rings, Interscience Tracts in Pure and Appl. Math., vol. 13, Interscience, New York, 1962. MR 27 \#5790.

9. S. Schafer, Introduction to non associative algebras, Academic Press, New York, 1966.

10. M. F. Smiley, The radical of an alternative ring, Ann. of Math. (2) 49 (1948), 702-709. MR 10, 6 .

11. O. Zariski and P. Samuel, Commutative algebra, Vol. II, University Series in Higher Math., Van Nostrand, Princeton, N. J., 1960. MR 22 \#11006.

DEPARTMENT OF MATHEMATICS, MICHIGAN STATE UNIVERSITY, EAST LANSING, MICHIGAN 48823 